'Departamento de Psiquiatría

y Salud Mental, Facultad de

Medicina, Universidad de

Concepción. Concepción, Chile.

aphD.

Trabajo no recibió apoyo financiero.

Los autores declaran no tener conflictos de interés.

Recibido el 14 de agosto de 2019, aceptado el 26 de mayo de 2020.

Correspondencia a: Gonzalo Navarrete Ríos. Avda. Juan Bosco s/n. Servicio de Psiquiatría - Departamento de Psiquiatría y Salud Mental. Casilla 160-C. Facultad de Medicina. Universidad de Concepción. Concepción, Chile. gonavarrete@udec.cl

\section{¿Es suficiente la base moral de los médicos?}

\author{
GONZALO NAVARRETE R.1,a
}

\section{Moral judgment of physicians is insufficient as a framework for medical ethics}

Physicians values are largely supported by a socio-cultural moral basis, also known as "classical utilitarianism". Technological advances and social questions to physicians show their paucity of an ethical conceptualization in medicine. A new way of approaching ethical conflicts in medicine should be constructed. Training should promote ethical reflection about these conflicts and about the actions of physicians. Ontogenetic and phylogenetic research on human nature, and the advances in moral psychology, could allow us to understand the construction of our judgment of values. An introspective emotional and rational effort to understand "how we are" and from there, to "how we act" lacks among physicians. This issue is even more complex in a political-social model which does not stimulate this type of analysis. The university space is a privileged opportunity to educate. The student must be envisioned as a human being whose professional acts should consider the needs of our society, aiming at a new ethical conceptualization in medicine.

(Rev Med Chile 2020; 148: 684-688)

Key words: Ethics; Emotions; Medicine.
A un cuando desde el inicio de nuestra formación médica se nos señala que los pilares que sustentan el edificio de nuestro quehacer valórico se basan en la épica del desarrollo de una humanidad justa, incorrupta, sacrificada y prudente, a través del perfeccionamiento individual en un contexto colectivo, es en la práctica profesional donde dicha construcción comienza a vislumbrarse de manera más nítida.

Nuestra base valórica previa al pregrado, y el desarrollo informativo -en cuanto recepción de información- y formativo-actitudinal que se da en el conocimiento, sociedad y la cultura ${ }^{1}$ parte de la vida universitaria, constituyen los cimientos del quehacer médico, enseñado por siglos, y con base en el juramento hipocrático.

Sin embargo, toda esa estructura valórica recepcionada por el médico que llevará a una conceptualización ética, se corresponde con el llamado utilitarismo moral clásico, que no está logrando satisfacer las necesidades de la población cada vez más instruida y consciente de sus derechos, y en cierta medida, de sus deberes.

En el contexto actual parece crucial el reflexionar, no tan solo desde las recomendaciones del discurso legal y/o sociocultural sobre el actuar del profesional, sino desde y hacia nosotros mismos.

El ser humano discierne en una escala de valores, aunque no siempre logrando una relación coherente entre su conciencia y actuar ${ }^{2}$. Nuestra individualidad, que incluye un "universo semántico" y emocional ${ }^{3}$, nos invita constantemente a reflexionar, en un gran marco de diversidad, contra siglos de una estructura valórica prestablecida que impide lograr una conceptualización ética en consonancia con nuestra naturaleza humana. Por ello, parece razonable plantear ¿qué está sucediendo actualmente con la ética médica? ¿qué camino debe seguir la profesión médica para 
resolver el reto qué nos propone la sociedad frente a la conceptualización ética?

Para responder estas preguntas, consideramos fundamental incluir el debate ético actual en medicina; el concepto de "naturaleza humana" desde una visión filosófica, biológica y psicológica para la construcción valórica; la relevancia de los procesos formativos en medicina para la conceptualización ética; y los avances en psicología moral, como base de una teoría para la conceptualización de la ética profesional.

\section{Moral y Ética Médica}

El delimitar el ámbito de la moral y la ética ha estado presente constantemente a lo largo de la historia, como plantea Ortiz Millán ${ }^{4}$ al enfatizar la importancia de distinguirlas para la filosofía moral. Este ámbito en particular se enmarcaría dentro del llamado "utilitarismo clásico", que se ocupa de satisfacer las necesidades del mayor número de individuos. Además, ofrece una teoría que entrega una orientación directa a la resolución de conflictos éticos, sobre la base de que los intereses de muchos, superan los intereses del individuo. Esto plantea un debate que podría requerir consideraciones basadas en la justicia y no tan solo en los derechos 5 .

Por años la "investigación filosófica de la moral”, se asumió estaba muy cerca de presuponer que la conducta moral incluía la reflexión ética. Es decir, se asumía que existía un cálculo moral utilitarista que incluía un proceso reflexivo íntimo que manifestaba una posición ética en la sociedad. Estos eran impuestos de forma autoritaria a todo el mundo, independientes del pensamiento y juicio humano ${ }^{6}$. Sin embargo, y si solo consideramos el componente individual, y por ende que los deberes éticos fuesen dictados de la conciencia, tampoco lograrían trascender a las más diversas acciones en la humanidad, porque la conciencia si no va referida a una materia precisa, es una mera referencia confusa ${ }^{7}$. Estos procesos reflexivos incluyen razonamiento pero, también intuiciones morales, las cuales son fundamentales reconocer ${ }^{8}$. Hume creía incluso, que la razón era (y solo podía ser) sirviente de las pasiones ${ }^{9}$. En resumen, y en palabras de Savelescu et al. ${ }^{10}$, el relativismo moral es falso, pero la ética implica sensibilidad al contexto y es dependiente de los hechos.
Si las consideraciones sobre la reflexión ética presentan dificultades a nivel individual, el enorme desarrollo científico y médico ya supera nuestra capacidad para responder las preguntas éticas que se nos proponen. Incluso Savelescu et al. ${ }^{10}$ plantea revertir el viejo aforismo ¿poder? y elevarlo a la pregunta ¿deberíamos?. El mismo autor reflexiona en el nuevo panorama ético, en donde el empoderamiento de los pacientes a través de internet es clave. Es decir, estas manifestaciones valóricas de los médicos que por décadas, sino siglos, no eran discutidas por el paciente, ahora son puestas en duda, e incluso, confrontadas.

Si llevamos el foco de análisis hacia la ética médica, aparece un interesante y actual debate. El juramento hipocrático como conjunto de principios éticos atemporales, tiende al interés de la salud del paciente y del gremio ${ }^{6}$, es decir, no considera los intereses del individuo, en esencia. El concepto de ética en medicina se afianzó a través de la historia convirtiéndose en transcultural, transreligioso y transnacional ${ }^{11}$. Pero, en las últimas décadas los cambios en nuestra sociedad nos impulsan al debate ético, donde la tecnología abre opciones ${ }^{10}$, al igual que la ciencia y una población con mayor acceso a educación. Es así como se abre un escenario de reflexión ética nuevo y que requiere aceptación. Savelescu et al. ${ }^{10}$ recoge esta realidad de manera precisa al decir, "donde no hay opciones, no hay preguntas éticas". Aparentemente habría consenso en que la ética médica, como señala R. Rhodes ${ }^{13}$, no puede ni debe derivarse de la moral común, es decir la medicina es una profesión y como tal, sus miembros tienen que internalizar y respaldar una ética específica ${ }^{12}$. Pero, $\mathrm{Holm}^{12}$ pone en duda la idea de R. Rodhes, de que la ética médica sea conceptualizada como ética profesional, y por ende, quede el contenido dejado a la profesión médica para desarrollar y decidir. Este aspecto sin lugar a dudas es un tema interesante de analizar, pero escapa al interés de la presente publicación.

Frente a un escenario que nos invita a replantearnos la construcción de las reflexiones éticas en medicina, es interesante considerar los aspectos esenciales de lo humano, tanto a nivel de procesos psíquicos como a nivel social. Si consideramos los factores racionales, individuales y socioculturales, que en su mayoría son construcciones post hoc creadas para objetivos estratégicos ${ }^{8}$, como los únicos elementos para dar una base moral y alcanzar una reflexión ética, estaríamos obviando 
la naturaleza humana ${ }^{14}$ y un componente intuitivo el cual incluso, es previo al razonamiento. La evidencia actual señala que la conducta moral presenta una base filogenética y ontogénetica ${ }^{14}$, y que las intuiciones estudiadas por la psicología moral, tienen un respaldo en las neurociencias, demostrándose una activación en áreas cerebrales relacionadas con la emoción ${ }^{15,16}$.

Con una sociedad más instruida y exigente, además de los avances en ciencias, podemos dimensionar la gran responsabilidad del reto hacia las reflexiones éticas de la medicina. Por ende, frente a la necesidad de iniciar un tránsito hacia una reflexión acorde a las necesidades actuales, que considere aspectos de la "naturaleza humana"14, tanto para el médico, como para el paciente, la opción de centrar nuestra mirada a la estructura emocional, como medio para intentar comprender la construcción de nuestros juicios valóricos, podría se el camino. Tema que lleva varios años siendo foco de estudio para la psicología moral ${ }^{8}$.

¿Y si estas reflexiones fueran canalizadas hacia la etapa académica de nuestros médicos?, ¿podríamos esperar una conceptualización ética distinta en medicina? ¿Qué ocurre en esta etapa? ¿Estamos considerando solo los procesos racionales y no incluimos nuestras intuiciones?

\section{Ética médica y Deontología.}

Los procesos formativos en medicina han ido de la mano de la tecnología y tendiendo a satisfacer las necesidades del modelo político-social. La relación educativa, aunque es evidentemente asimétrica ${ }^{17}$, permite la transmisión de conocimiento y valores desde el docente. El estudiante tiene la posibilidad de ejercer un proceso reflexivo no tan solo racional, en tanto la temática en particular, sino que además desde lo ético. Por ende, quizá la pregunta inicial al revisar nuestros modelos educacionales y "formativos" es ¿a nuestros alumnos los estimulamos a realizar reflexiones éticas o solo los hacemos partícipes del modelo utilitarista moral clásico?.

Esta oportunidad de formar de manera global a un médico es considerado, según Betancur ${ }^{18}$, como un espacio privilegiado donde se congrega en torno al saber y desde allí es posible plantear experiencias para el hacerse a partir del Ser. El ser profesional no es ser alguien, se es alguien para hacerse profesional. Al parecer existe una escisión entre ser persona y ser profesional; es por ello fundamental replantear la escisión entre ser y hacer; no hay una ética de las profesiones escindida de la ética del sujeto, no es posible pensar al estudiante solo como profesional en formación, es un imperativo pensarlo como un ser para el hacer.

Ramos y Bedregal ${ }^{19}$, señalan la necesidad de educar el juicio moral de los futuros médicos, en una comunidad universitaria en que los valores se vivan, motiven y desarrollen. Plantean que el nuevo contexto del ejercicio de la medicina demanda una nueva alianza terapéutica, como así mismo configurar las garantías y límites de la libertad del paciente y del médico. Pero, estas consideraciones establecen la necesidad de mirar más allá de nuestra argumentación valórica, siendo este reto, recogido por $\mathrm{Haidt}^{8}$ al preguntarse ${ }^{2} \mathrm{Cuan}$ difícil puede ser enseñarle a los estudiantes a mirar siempre al otro lado, a buscar siempre evidencia que contradiga su punto de vista predilecto?. Parece ser muy complejo si no logramos entender el importante peso emocional en nuestros juicios morales. Podría ser incluso necesario el uso de instrumentos para lograr una conceptualización ética ${ }^{20}$.

De lo anterior, se desprende que la tarea formativa es gigante, sobre todo cuando la emancipación de la economía, en palabras de Bauman ${ }^{21}$, ha roto las ataduras políticas, éticas y culturales, de la vida social. Es así como la responsabilidad, tanto del médico como del paciente, podría tener una rol crucial. Al médico le corresponde no tan solo procurar la salud del individuo, sino que considerar la opinión éste. Pero, si el paciente no asume su autocuidado con responsabilidad, por ejemplo, tanto en forma retrospectiva como prospectiva, se genera un conflicto no fácil de resolver, por ende, en esa instancia ¿Es momento para que el médico tome un papel distinto?5.

De lo anterior, nace la tarea como docentes de lograr en nuestros alumnos, no solo que "declaren" que son conscientes del momento de la humanidad. No podemos pretender que asignaturas centradas en el análisis teórico y práctico de la ética médica, que lo único que intentan es perpetuar el utilitarismo moral, logren lo que no hemos podido lograr en siglos. Debemos incentivar a nuestros estudiantes a entender que los conceptos éticos en medicina requieren de una mirada crítica, sin 
ataduras, en donde el centro del análisis esté en el paciente y desde la profesión médica.

\section{El acto médico}

La interacción del médico - paciente, incluye un lenguaje verbal y no verbal, que denota una base intelectual, emocional e incluso espiritual, las cuales van inherentemente unidas, aunque alguno de los participantes de esa comunicación lo evite. Esta interacción, pretende un equilibrio comunicacional entre el actuar del médico, y considerando los mismos componentes, en su independencia y variabilidad, quien padece. Es decir, el médico debiese partir, como plantea Maturana $^{22}$, "aceptando la legitimidad del otro" para iniciar este tipo de relación humana. Este paso podría ser clave para avanzar en la conflictiva ética, si además los profesionales comprendieran que la "ética" podría ser una ayuda acerca de qué debemos hacer y qué no hacer en esta interacción, y no entenderla como burocracia ${ }^{10}$. Esta interacción y necesidad de legitimización del otro podría tener un camino, como señala Haidt ${ }^{8}$, en donde el desarrollo humano va de la mano de intuiciones morales, las cuales emergen en edades tempranas. Debemos ser conscientes de que somos criaturas intuitivas cuyos instintos impulsan nuestro razonamiento estratégico.

Considerar estos elementos en el perfeccionamiento formativo de los médicos, podría ayudar a una construcción del concepto de ética más apropiado a las necesidades de nuestros pacientes, además de incluir, como plantea $\mathrm{McCullough}^{6}$ una vigilancia de médicos y líderes de la profesión, sobre la integridad profesional. Pero, no dejando fuera en nuestra opinión, a quien padece o pudiese representar sus intereses.

La medicina necesita profesionales que se distingan por su fortaleza para generar una disciplina moderna y eficiente pero, de la mano de una base axiológica sólida. Las enseñanzas del pregrado nos deberían llevar a un mundo abundante en conocimiento teórico-práctico, pero además al autodescubrimiento, lo cual debiese potenciarse en nuestra práctica profesional y/o en la formación como especialistas. Es así como preparamos nuestras conciencias para ajustar nuestras normas más íntimas (ética) y comportarnos "adecuadamente" dentro del marco histórico-social (moral). Este desarrollo reflexivo-ético, siendo conscientes de nuestra fragilidad de ética profesional en medici$\mathrm{na}^{6}$, nos abre las ventanas del mundo y nos llama a la acción, pero a una acción empática, sensata y efectiva.

\section{Conclusiones}

Del análisis de la moral, la ética y el quehacer médico, se desprende en gran medida lo que creemos se espera de quien abraza la medicina. La orden médica, se ha centrado favorablemente en construir un ética centrada en la rectitud, la prudencia y la filantropía. Sin embargo, a la luz de los avances en la ciencia y los cambios en la sociedad, la conflictiva en medicina plantea que las reflexiones éticas no están logrando reflejar las necesidades de nuestros pacientes en el resultado, en quien la dirige y en qué términos. Durante siglos, el llamado utilitarismo moral ha intentado ordenar y representar los intereses del gremio médico y los pacientes, pero es evidente que ello no se ha logrado. Nuestra sociedad está pidiendo cambios en muchos aspectos, y la conceptualización de una ética médica no es la excepción. El "querer querer" como un acto de voluntad que debe partir con una actitud para generar un cambio en nuestro sentir y actuar, amerita un trabajo sobre nuestra consciencia, un autodiagnóstico, y una observación continua de nuestra emocionalidad y raciocinio.

Las abundantes publicaciones sobre ética médica y el imperativo de adaptar la medicina a las necesidades de la sociedad, nos obliga a reconsiderar nuestra conceptualización en ética. De ahí que la investigación filogenética, ontogenética y en psicología moral, podría ser un primer paso importante hacia este objetivo.

Paralelamente, requerimos reenfocar la docencia en nuestras universidades, no es posible concebir que nuestros médicos logren un desarrollo valórico, sin un curriculum que estimule el desarrollo formativo, y no meramente informativo, como es impresión de lo que ocurre actualmente. Necesitamos instituciones y médicos formadores que estimulen a la reflexión y autoobservación en el alumnado, mediante la entrega de conocimientos teóricos-prácticos, pero también con una base clara sobre los aspectos filosóficos, biológicos y psicológicos que influyen en nuestras construcciones valóricas. Es evidente que el objetivo de lograr 
una base teórica para la conceptualización de la ética profesional, y en particular, de la medicina, es compleja. Pero, consideramos que estas líneas de investigación podrían ser el camino adecuado.

Agradecimientos: Agradezco al apoyo, guía y constante invitación a la reflexión crítica por parte de mis maestros, Dr. Rolando Pihán V., Dr. Benjamín Vicente P., Dr. Pedro Rioseco S. y de manera muy especial a quien ya no está con nosotros, el Dr. Mario Muñoz R.

\section{Referencias}

1. Ortega y Gasset, Misión de la Universidad. Editorial Cátedra. 2015.

2. Ferrater Mora. Diccionario de Filosofía. J. Ed. Ariel. 1999.

3. Holbach, Baron. La moral universal o los deberes del hombre fundados en su naturaleza. 1812.

4. Ortiz Millán G. Sobre la distinción entre la ética y moral. Isonomía no 45, octubre 2016, pp. 113-139.

5. Pugh, J. Navigating individual and collective interests in medicla ethics. Journal of Medical Ethics 2018; 44: 1-2.

6. McCullough LB. The ethical concept of medicine as a profession discovery or invention? J Med Ethics 2019; 45 (12): 786-7.

7. Aristóteles. Ética a Nicómaco. [siglo V a. C.]. Universitat de València. 1993. ISBN 9788437014029.

8. Haidt J. La Mente de los Justos. Barcelona, España: Editorial Planeta, 2019.

9. Mellizo, Carlos. Investigación sobre los principios de la moral, Alianza, Madrid, 2014.

10. Savulescu J, Douglas T, Wilkinson D. Philosophical medical ethics: more necessary than ever. J Med Ethics 2018; 44: 434-5. http://dx.doi.org/10.1136/medethics-2018-104993.

11. McCullough LB, Coverdale JH, Chervenak FA. Profes- sional ethics in obstetrics and gynecology. NewYork, NY, and Cambridge, UK: Cambridge University Press, 2020; 1-23.

12. Holm S. Roles, professions and ethics: a tale of doctors, patients, butchers, bakers and candlestick makers. J Med Ethics 2019; 45: 782-3. http://dx.doi.org/10.1136/ medethics-2019-105826.

13. Rhodes R. A Defence of medical ethics as uncommon morality. J Med Ethics 2019; 45: 792-3. http://dx.doi. org/10.1136/medethics-2019-105941.

14. Ardilla R. Filogénesis y ontogénesis de la moral. Rev Acad Colomb Cienc 2014; 38: 205-15.

15. Helion Ch, Ochsner K. The Role of Emotion Regulation in Moral Judgment. Neuroethics 2018; 11 (3): 297-308; A Neural Network for Moral Decision Making. http:// dx.doi.org/10.1016/B978-0-12-397025-1.00180-9.

16. Han H, Chen J, Jeong CH, Glover G. Influence of the cortical midline structures on moral emotion and motivation in moral decision-making. Behav Brain Res 2016; 302: 237-51. https://doi.org/10.1016/j. bbr.2016.01.001.

17. Amilburú M, Ruiz-Corbella M, García-Gutierrez J. Intervención Sociocomunitaria (2011). Capítulo: Ética y Deontología profesional en la Intervención Sociocomunitaria. Ed. G. Pérez Serrano, pp. 465-86.

18. Betancur GE. La ética y la moral: paradojas del ser humano. Revista CES Psicología 2016; 9 (1): 109-21.

19. Ramos Vergara P, Bedregal García P. La ética médica en discusión: análisis de los principales temas sobre ética médica en la práctica clínica tratados en revistas nacionales de medicina y bioética 1998-2013. Acta Bioethica 2017; 23 (1): 71-81.

20. Svantesson M, de Snoo-Trimp JC, Ursin G, et al. Important outcomes of moral case deliberation: a Euro-MCD field survey of healthcare professionals' priorities. J Med Ethics 2019; 45: 608-16.

21. Bauman, Z. Modernidad Líquida. Fondo de Cultura Económica. 2016.

22. Maturana Romesín, H. El Sentido de lo Humano. Editorial JC Saéz. 2008. 